\section{Especialidades derivadas de la cirugía general: revisión de brechas e intención de residentes de la especialidad en Chile}

\author{
EUGENIO GRASSET ${ }^{1, \mathrm{a}}$, MIGUEL OBAÍD ${ }^{1, \mathrm{~b}}$
}

\section{Gaps between country needs and intentions to obtain a subspecialty among general surgery trainees in Chile}

The fragmentation of a general specialty in subspecialties or derived specialties is a widely spread reality. Chilean health care system is becoming more complex, requiring more specialists. On the other hand, doctors in specialty training increasingly choose a subspecialty to continue their training and professional development. This contrasts with the growing need for well-trained general surgeons. We aimed to compare the evidence about the needs for general surgeons and the perspectives of Chilean physicians about their specialty training. A literature review about the intention of specialization in Chilean general surgery residents and the gaps in the Chilean health system, was performed. As of December 2016, there were 2,103 general surgeons in Chile, of whom 598 (28\%) also have a subspecialty. Among the latter, $49 \%$ are plastic or vascular surgeons, which are also the specialties with the greatest demand in the public system. According to estimates of the Chilean Ministry of Health, on that year there was a deficit of 285 general surgeons and 142 subspecialists. These figures correspond to $18.5 \%$ and $23.8 \%$ of the existing resources. A survey published in 2009 reported that $78 \%$ of trainees in general surgery would prefer to continue studying a subspecialty, following the trend observed in the USA and Europe. Therefore, there is a disproportion between the intentions of general surgery trainees and the needs for these professionals in Chile.

(Rev Med Chile 2018; 146: 1325-1333)

Key words: General Surgery; Internship and Residency; Specialties, Surgical; Surgeons.
'Departamento Cirugía General

Hospital del Salvador, Universidad de Chile. Santiago, Chile.

aMagíster en Educación en

Ciencias de la Salud.

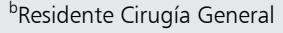

Hospital del Salvador, Universidad

de Chile, Magíster en Cs.

Biomédicas Mención Morfología.

Los autores no recibieron apoyo financiero ninguno para la presente publicación y declaran no tener conflictos de interés.

Recibido el 20 de noviembre de 2017, aceptado el 25 de septiembre de 2018.

Correspondencia a: Eugenio Grasset Av. Salvador 364, Providencia. Santiago, Chile. ea.grassete@uchile.cl

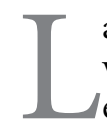

a cirugía general se ha fragmentado cada vez más en especialidades derivadas y las enfermedades previamente resueltas por cirujanos generales ahora son tratadas por estos especialistas en lo que parece responder a una evolución propia del avance técnico de la especialidad, desdibujando el área de desempeño de los cirujanos generales ${ }^{1}$. Por lo mismo, no es de extrañar que en los Estados Unidos de Norteamérica entre 70 y $80 \%$ de los cirujanos egresados elijan continuar su formación en alguna área específica de la especiali- dad, fenómeno que ha llevado a una disminución de $26 \%$ en el número de cirujanos generales en este país entre los años 1980 y el 2005². Por otra parte, debido al crecimiento de la población senescente y de la demanda de servicios médicos, se estima que la necesidad de cirujanos generales aumente en $25 \%$ para el 2025, pero de acuerdo a la proyección actual, se estima que el número de cirujanos que practique la cirugía general disminuirá en $18 \%$ adicional los próximos 20 años 2 .

En Chile, disponemos de escasa información 
respecto al desarrollo profesional de los cirujanos generales y de las intenciones de parte de los formandos en cuanto a su carrera profesional. Si bien parece haber un acuerdo global sobre la evolución local de la profesión de cirujanos generales hacia una mayor especialización, no sabemos si eso se condice con las necesidades del sistema sanitario nacional ni de la población.

Este estudio desarrolla un primer análisis de un problema no tratado en nuestro medio, a través de una revisión de la evidencia actual sobre la brecha existente entre el número de cirujanos generales y de especialistas (con especialidad derivada) en nuestro país, cuáles son los distintos programas de especialidad, cuántas y cuáles universidades las imparten, las preferencias de los residentes entre especialización o continuar su ejercicio como cirujano general así como la proyección de la demanda en Chile.

\section{Método}

Se realizó una revisión de la evidencia disponible por medio de una búsqueda en las plataformas de Pubmed, Scielo y Biblioteca Virtual del Ministerio de Salud (MINSAL) (http://www. bibliotecaminsal.cl/) con los términos de fellowship, training, subspecialization, surgical education, surgical residency, residencia, beca, especialización, subespecialización, especialidad derivada, brecha $y$ educación médica además de buscar en la biblioteca digital de la Superintendencia de Salud (SIS). Para obtener los datos desde las Universidades se accedió al Sistema de Información de Educación Superior (SIES) del Ministerio de Salud (MINEDUC) de la que no se obtuvo información en detalle, por lo que se accedió a cada Universidad para extraer los datos de sus programas o mallas publicadas. El presente artículo es el resultado de esta revisión y del análisis de los datos obtenidos con estadística descriptiva.

\section{Realidad internacional}

En Estados Unidos de Norteamérica el número de residentes de cirugía que eligen la práctica de cirugía general ha disminuido a medida que ha aumentado la popularidad de las especialidades derivadas. Actualmente, entre 70 y $80 \%$ de los residentes de cirugía general optan por una de éstas. Algunos autores explican esta tendencia dado que $85 \%$ de los residentes al final de su formación se consideran novatos respecto al trabajo independiente, la documentación necesaria para el mismo y codificación del mercado ${ }^{3}$. Al principio pareció ser solo un tema de complejización de la técnica, pero es probable que esto responda a causas multifactoriales las que se resumen en la Tabla 1. De éstas cabe destacar que más allá del evidente avance de la técnica, de la necesidad de adiestramiento en nuevas tecnologías y de la profundización en el conocimiento de determinados sistemas, se agregan los intereses individuales de focalización, ya sea en poblaciones particulares o en patologías de manejo multidisciplinario o en determinados órganos y sistemas. Asimismo, resulta interesante mencionar la importancia del contexto formativo, particularmente el currículum y los centros formadores de cirujanos generales, cada vez más complejos y alejados del ejercicio de la especialidad general. Y por último, los incentivos estructurales y del mercado, como retorno pecuniario, desarrollo de la carrera profesional o posibilidad de acceder a determinadas instituciones de prestigio $^{1}$ (Tabla 1 ).

Actualmente, existe una mayor amplitud de conocimientos y procedimientos, tales como técnicas de laparoscopía avanzada, endoscopía, cirugía endovascular, robótica, entre otras, además de la patología de manejo multidisciplinario. Además se observa una percepción generalizada de

\section{Tabla 1. Incentivos a la formación en especialidades derivadas}

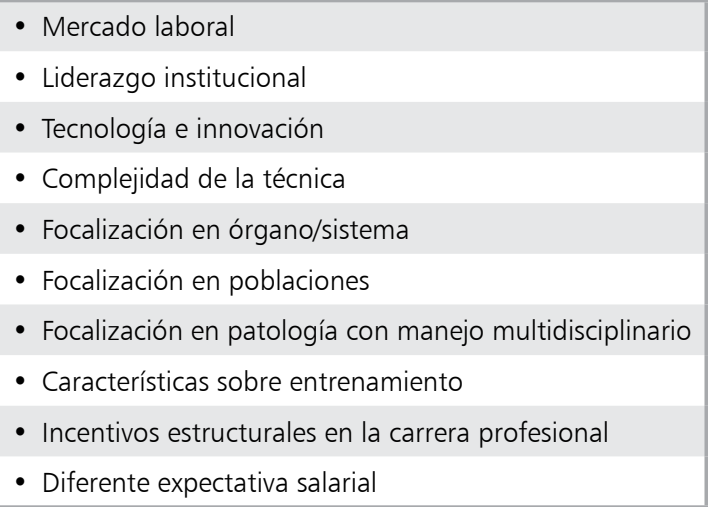

Modificado de Bruns SD y cols. J Gastrointest Surg. 2014; 18 (8): 1523-31. 
que muchos graduados carecen de las habilidades necesarias para realizar con seguridad algunas operaciones laparoscópicas básicas y muchas de las avanzadas. Los programas de especialidades derivadas tienen el potencial para cerrar estas brechas encontradas en muchas residencias, proporcionando al cirujano graduado una oportunidad de dominar las habilidades quirúrgicas, ganar confianza y autonomía en forma progresiva. La experiencia extra también facilita la transición de la formación a la práctica independiente ${ }^{4}$.

Desde el punto de vista formativo, al menos en Estados Unidos de Norteamérica parece ser que tanto el ambiente como el currículum influyen en la elección laboral de los egresados. Por ejemplo, en aquellos programas con menos oferta de especialidades derivadas y programas de post-residencia egresan cirujanos que eligen en mayor medida el ejercicio como cirujanos generales. En contraste, por cada especialidad derivada presente en la institución, aumenta en $2 \%$ la proporción de residentes que eligen continuar estudiando. Por otra parte, la presencia de rotaciones rurales, en servicios de urgencia y en atención abierta también favorece la elección de cirugía general. Finalmente, no parece haber relación con tipo de programa (universitario o independiente) ni con el número de residentes ${ }^{2}$.

\section{Realidad en Chile}

Este ha sido un tema poco estudiado en nuestra región y en Chile particularmente. Para el estudio de las necesidades de especialistas se han utilizado distintos modelos y métodos desde el 2009 por el Banco Mundial ${ }^{5}$ y el MINSAL ${ }^{6,7}$. Dada las particularidades de nuestro sistema sanitario, la mayor cantidad de información disponible sobre necesidades de especialistas y oferta laboral es de los Servicios de Salud del MINSAL. El mercado privado no presenta datos agregados sobre la necesidad de especialistas o médicos y la única información disponible sobre privados es aquélla que viene del cruce de datos entre el Servicio de Impuestos Internos, la Corporación Nacional Autónoma de Certificación de Especialidades Médicas (CONACEM), el Fondo Nacional de Salud (FONASA) y el Sistema Informático de Recursos Humanos del Ministerio de Salud (SIRH) ${ }^{8}$.

La OPS estableció en el 2006, en el Llamado a una Década de Recursos Humanos de Toron- to, que debajo de una densidad mínima de 25 profesionales por 10.000 habitantes no se puede conseguir el acceso de toda la población a prestaciones de salud básicas ${ }^{7}$. Y para Chile, el Informe del Banco Mundial del 2009 fue el primer estudio en determinar la brecha de manera confiable.

La última publicación al respecto de la "Organisation for Economic Co-operation and Development" (OECD) del año 2017 señala a Chile con la más baja densidad de médicos (21 por 10.000 habitantes) junto con Corea y Turquía, versus el resto de los países de la OECD, con un promedio de 34 médicos por cada 10.000 habitantes versus 21 por 10.000 en Chile. Incluso a nivel regional estamos bajo otras naciones tales como Argentina (39 por 10.000 hab.), México (24 por 10.000 hab.) y Uruguay (37 por 10.000 hab.), que se encuentra en una situación cercana a la de Brasil ${ }^{7,9}$.

Según los indicadores de la $\mathrm{OMS}^{10}$, del total de 38.658 médicos en Chile, 53,7\% son especialistas versus $88 \%$ en Estados Unidos de Norteamérica, 50-56\% para Canadá, Holanda y Francia, lo que puede estar explicado por las diferencias en el modelo sanitario de cada país, correspondiendo a $62 \%$ el promedio de los países de la OCDE.

Actualmente, existen distintas opciones y horizontes para el residente de cirugía general graduado, considerando que algunas de ellas no son reconocidas por el MINSAL y que también no son exclusivos de cirugía (Figura 1). A diciembre de 2016 hay inscritos 2.103 cirujanos en la Superintendencia de Salud (SIS), de los cuales 598 registran además una especialidad derivada, lo que se resume en la Figura 2, donde es importante considerar que algunas de las especialidades como cirugía digestiva no se registran en la SIS ya que al momento de la revisión no era una especialidad reconocida. Llama la atención que cirugía plástica y vascular agrupan $48,3 \%$ de las preferencias ${ }^{6}$ (Figura 1 y 2 ).

\section{Brechas en Chile}

Existen distintos factores que influyen en la brecha de especialistas y cirujanos generales en nuestro país. Estos están dados, entre otros, por:

\section{Ingreso de profesionales}

- Inmigración.

- Formación.

- Certificación. 


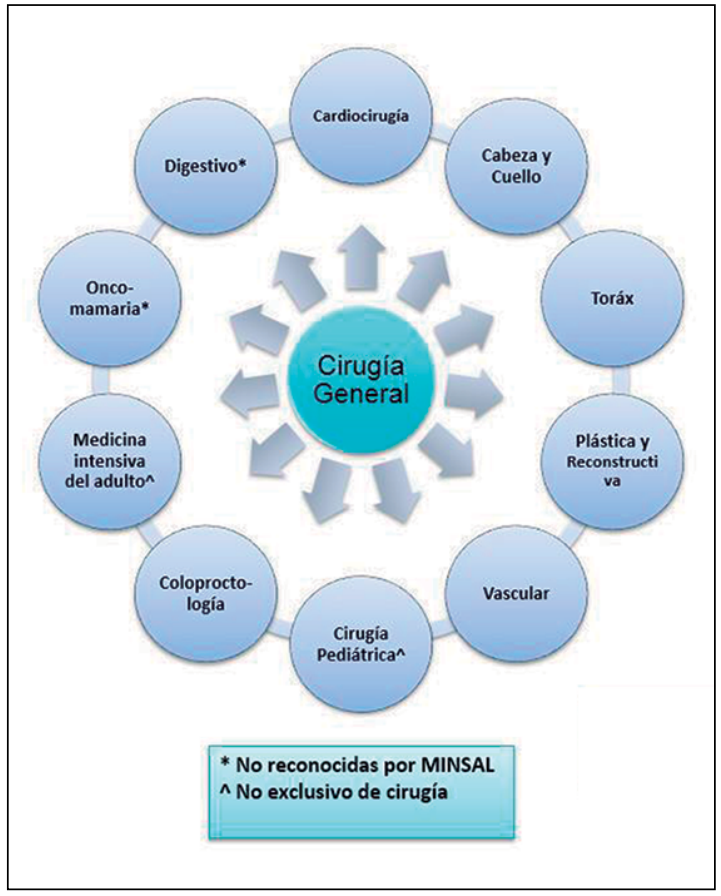

Figura 1. Opciones de especialidad del residente en cirugía general una vez graduado. Se describen disciplinas no reconocidas por el MINSAL como otras que no son exclusivas de la especialidad de cirugía. Confección propia con datos de $\mathrm{e}^{13}$.

\section{Aumento de demanda}

- Cartera de inversión hospitalaria.

- Crecimiento poblacional.

- Migración entre sistema público/privado.

- Cambio en prestaciones (densidad y tipo) otorgadas.

\section{Salida de profesionales}

- El retiro incentivado desde la autoridad central.

- Jubilación.

- Fallecimiento.

- Migración.

Por otra parte, si analizamos la brecha nominal declarada por la Subsecretaría de Redes del MINSAL en su informe de brechas de 2016 (el del año 2017 no considera las brechas de sub-especialistas $)^{7}$ vemos algunos cambios: cirugía vascular es la sub-especialidad que presenta la mayor brecha con 55 profesionales en déficit lo que representa a $51,8 \%$ de los actualmente inscritos; luego aparece cirugía plástica con 30 profesionales en déficit, lo que representa $23,8 \%$ de los actualmente registrados. Si consideramos el déficit de cirujanos generales, con 1.540 inscritos, se requieren 285 adicionales, un déficit de 18,5\%. Las Figuras 3 y

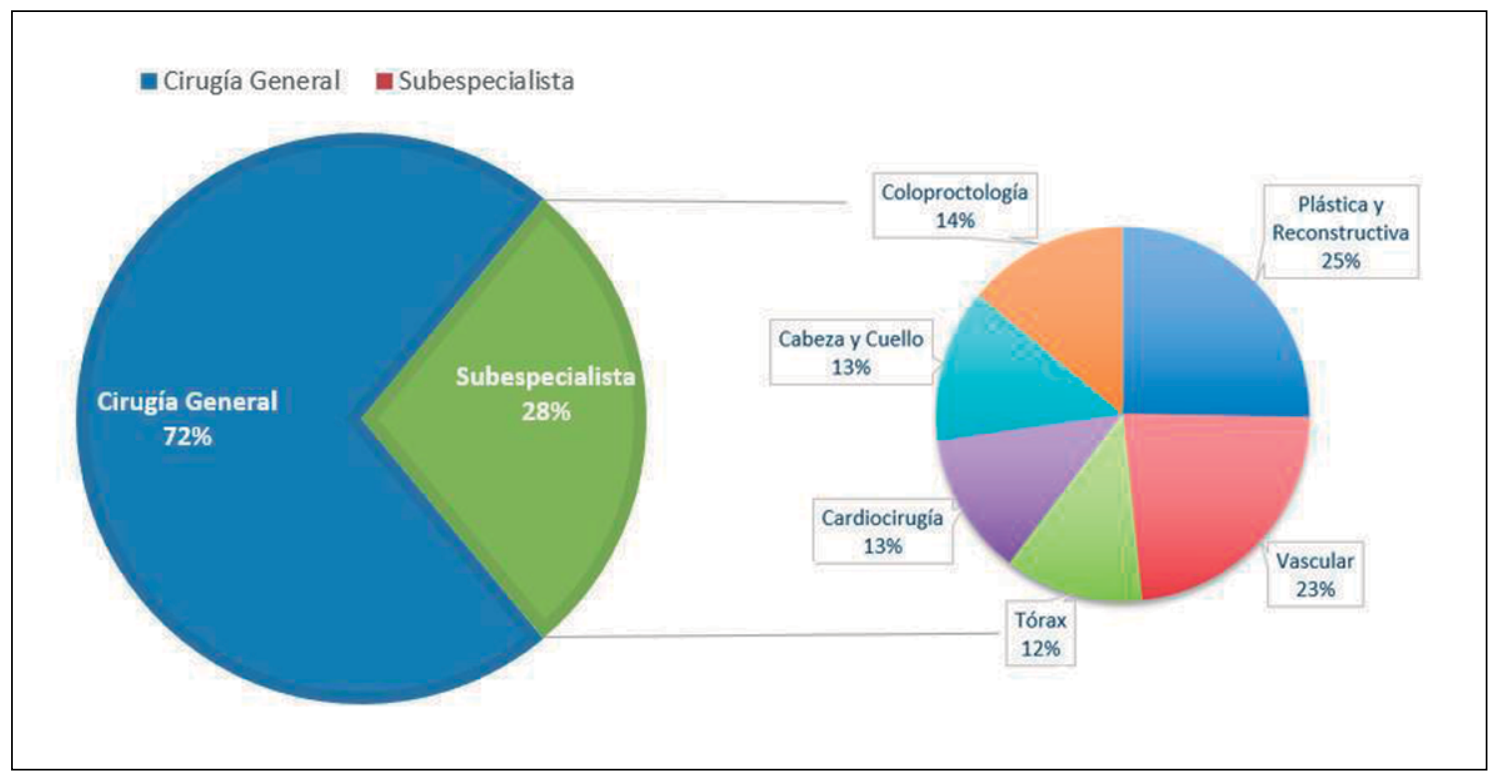

Figura 2. Cirujanos inscritos en Superintendencia de Salud (SIS). Confección propia con datos de ${ }^{6}$. Dato de especialidad de Digestivo Alto o Cirugía Digestiva no figura ya que la especialidad no estaba reconocida como tal al momento del levantamiento de datos. 
4 muestran las brechas existentes en las distintas especialidades derivadas de la cirugía general (Figura 3 y 4 ).

La formación en práctica y certificación por CONACEM han significado un aumento en la inscripción de especialistas, asimismo los cambios migratorios de los últimos años, sumado a cambios regulatorios, como la autorización del ejercicio profesional en Chile por el mero reconocimiento de la especialidad. Es de esperar entonces, que estas consideraciones modifiquen aún más el escenario en los años por venir, pero no se encontraron datos que permitan realizar un pronóstico al respecto.
De todas maneras la principal vía de formación de profesionales de la salud en Chile sigue siendo la universitaria, con $83 \%$ de los médicos inscritos en la SIS ${ }^{6}$, lo mismo se cumple para las especialidades derivadas y es de estos programas universitarios de los que se dispone la mayor información. Las universidades que las imparten, son la Universidad de Chile, Universidad de Concepción, Pontificia Universidad Católica de Chile, Universidad de Valparaíso, Universidad de Los Andes, Universidad de Santiago, Universidad del Desarrollo y Universidad de Concepción (según los datos obtenidos en sus propias páginas web a diciembre del 2017). Si observamos lo anterior, destaca la
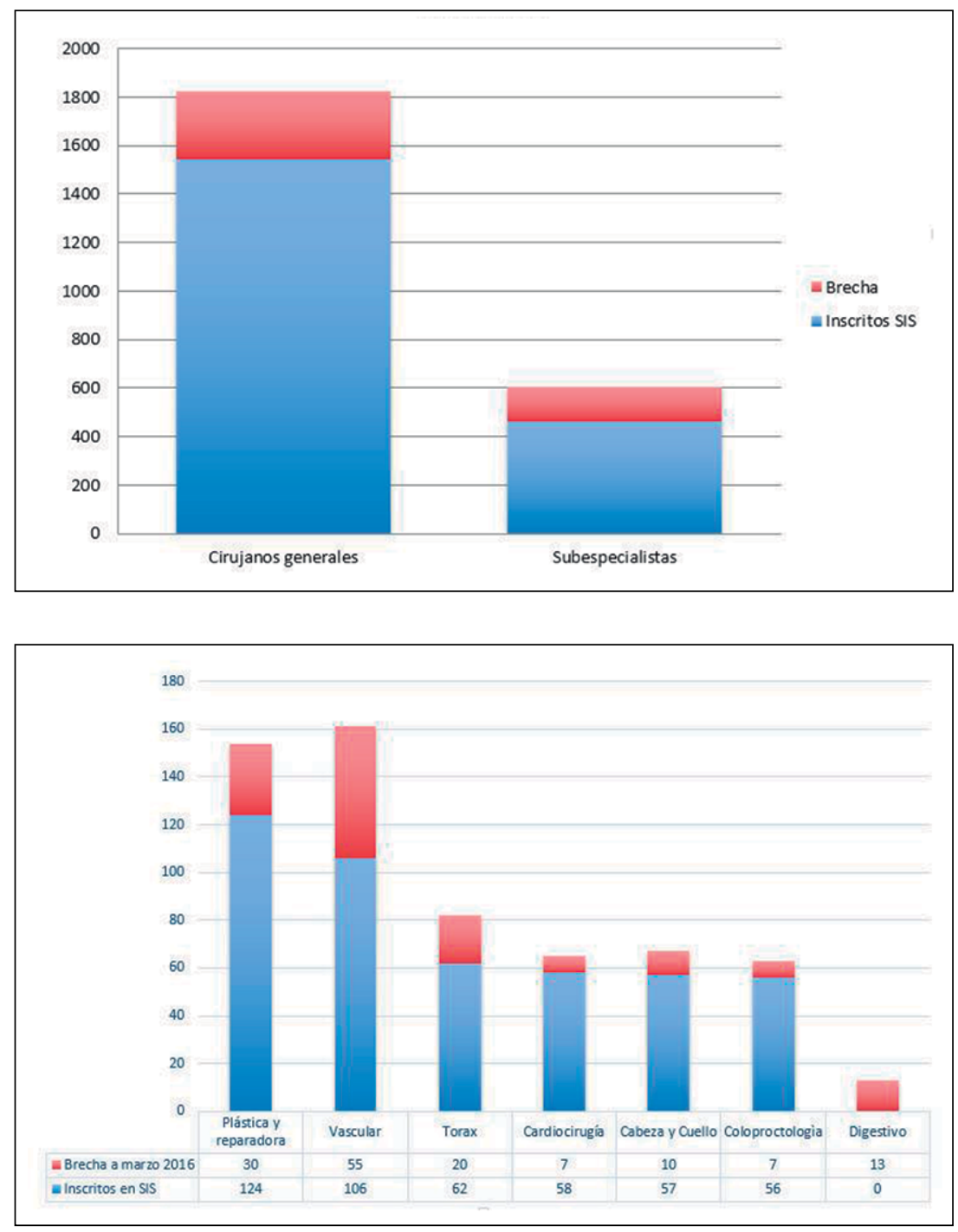

Figura 3. Brecha existente en relación a cirujanos generales y especialistas según la necesidad nacional confección a partir de ${ }^{7}$.
Figura 4. Cirujanos especialistas en déficit según la Subsecretaría de Redes Asistenciales. Confección propia de datos de? 
gran concentración de oferta formativa solo en la ciudad de Santiago, dejando para regiones solo la formación de cardiocirugía en Valparaíso y coloproctología y cirugía vascular en Concepción (información extraída de sus páginas web) (Tabla 2).

Finalmente, respondiendo la interrogante sobre qué es lo que los residentes y recién egresados desean hacer, la única información disponible es sobre un universo de 131 residentes de cirugía general encuestados el $2007^{11}$. De ellos, $27,3 \%$ pretende ejercer como cirujano general, de los cuales $62 \%$ se desarrollaría en regiones. El 72,7\% quiere seguir una especialidad derivada, de los cuales en su mayoría (70\%) la desarrollaría en Santiago. Siendo la más cotizada Cirugía Plástica y Reconstructiva y la de menor preferencia Trasplante $^{11}$ (Figura 5).

La aspiración de sub-especializarse luego de concluir la formación en cirugía general, es similar a lo ya mencionado por los residentes estadounidenses, y podrían corresponder a intereses similares, si bien las motivaciones particulares de la población nacional no han sido estudiadas dirigidamente.

Tabla 2. Base Oferta Académica Postgrados y Postítulo 2016

\begin{tabular}{|lll|}
\hline Santiago & Valparaíso & Concepción \\
\hline Cabeza y cuello & Cardiocirugía & Coloproctoctología \\
Cardiocirugía & & Vascular \\
Coloproctoctología & & \\
Digestivo & & \\
Oncología & & \\
Cirugía Plástica y Reconstructiva & & Universidad de Concepción \\
Tórax & & \\
Vascular & Universidad de Valparaíso \\
Universidad de Chile & & \\
Universidad del Desarrollo & & \\
Universidad de Santiago & & \\
Universidad de los Andes & & \\
Pontificia Universidad Católica de Chile & & \\
\hline
\end{tabular}

Distribución en la formación en las distintas especialidades derivadas de la cirugía general en distintas Universidades en Santiago y regiones. Confección propia a partir de información disponible en las web de postgrado.

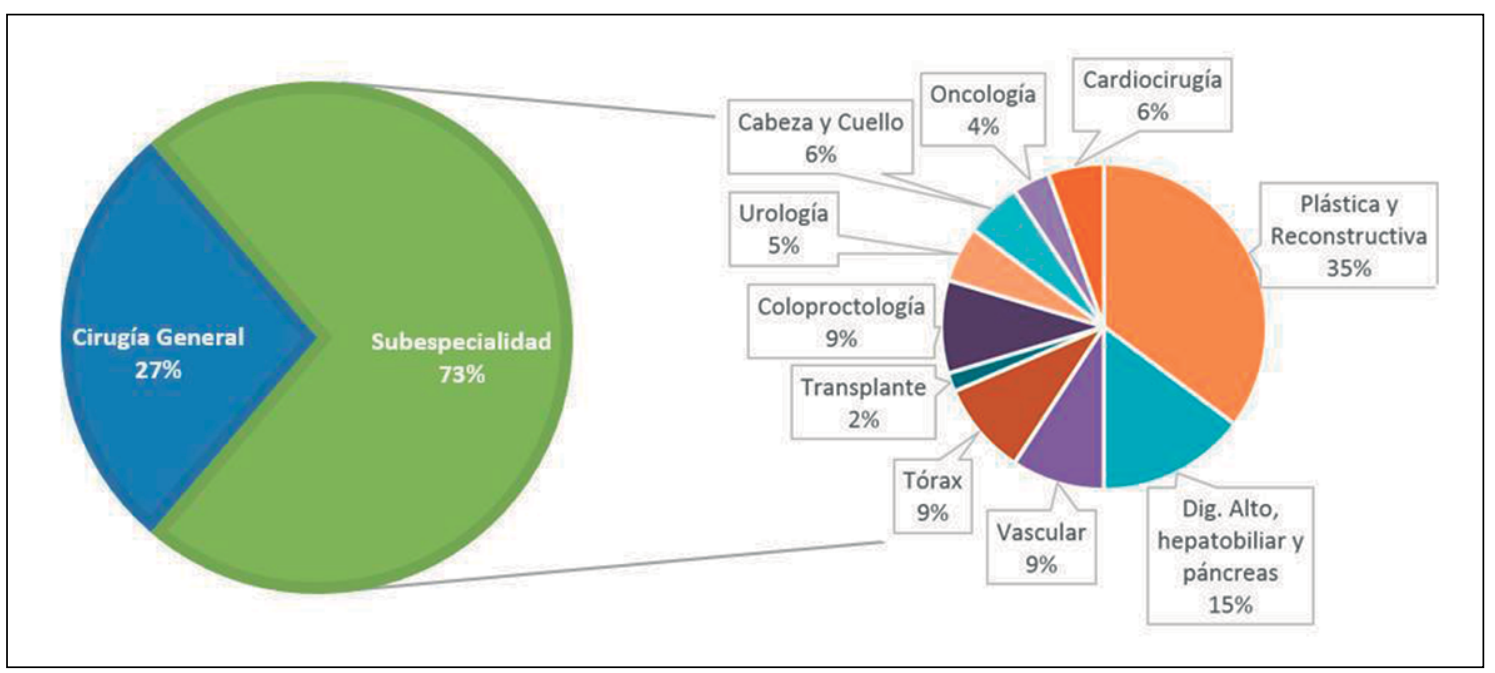

Figura 5. Preferencias de los residentes y recién egresados en cirugía general respecto a su formación. Confección propia a partir de datos extraídos de ${ }^{11}$. 


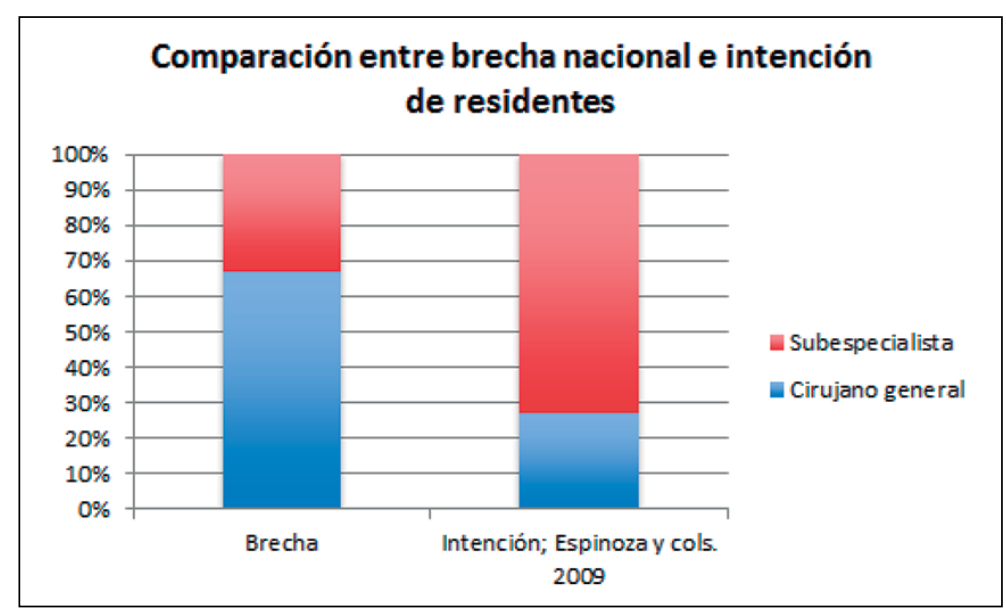

Figura 6. Diferencia entre intención de residentes de cirugía general y la distribución de la brecha de cirujanos generales y especialistas. Confección propia.
Finalmente, la información disponible sugiere que existe una diferencia entre las necesidades del país y la intención de los residentes, quienes presentan una tendencia en proporción mayor hacia elegir una especialidad derivada que la que representa la brecha entre cirujanos generales y especialistas (Figura 6).

\section{Proyección}

La proyección de la brecha es algo complejo de definir y depende de cómo se modelen la oferta de profesionales y la demanda sanitaria futuras, cada uno influido por múltiples factores, que escapan a lo meramente sanitario. De esta forma, los patrones de formación universitarios y cómo estos se adaptan a las necesidades sanitarias, la incorporación de profesionales desde otros países, así como políticas de incentivo al retiro y envejecimiento de los profesionales serán relevantes, por nombrar algunos ejemplos.

Una de las estrategias más ambiciosas ha sido la creación, por parte del MINSAL, del "Programa de formación y retención de especialistas”, el cual financia la formación de especialistas médicos en programas universitarios, les otorga un estipendio de mantención durante el curso de su residencia, con el compromiso que una vez egresados éstos retribuyan con un período obligatorio de devolución en el servicio público: el Período Asistencial Obligatorio (PAO). Actualmente se está en un momento de incertidumbre respecto a las políti- cas para especialistas en $\mathrm{PAO}$, ya que se exige un mínimo de dos años de devolución previa para poder postular a una especialidad derivada o a cualquier otro programa ${ }^{12}$. Asimismo, el médico en devolución estaría limitado al plan de desarrollo de la institución donde trabaja, por lo que la elección del área donde seguir formándose depende de aquello.

Si consideramos el escenario actual, pareciera que la capacidad formadora es insuficiente en algunas áreas; por ejemplo proyectando ningún retiro de cirujanos vasculares, estos recién verán satisfecha su brecha en 10 años a demanda similar a la actual.

Si contrastamos lo anterior con la concentración de especialistas en rangos etarios mayores, parece del todo pertinente la participación activa del estado para favorecer la formación de más especialistas. Al año 2015 6\% de la población médica tiene $>65$ años (la edad de jubilación para los hombres en Chile, 60 años para las mujeres) pero si revisamos del total de especialistas, 13,5\% está en edad de acceder a la jubilación (mayor a 65 años $)^{6}$. Por otra parte, si nos comparamos con los países del OECD, la proporción de médicos de más de 55 años en el 2013 alcanzaba un promedio de $33 \%$ en los países de la OCDE, mientras que en Chile era tan sólo de $28 \%$.

No se disponen de datos confiables sobre la matrícula de especialistas ni sobre los mecanismos de financiamiento o devolución a nivel nacional por lo que las proyecciones se basan sobre estimados y no es posible proyectar su evolución. 


\section{Conclusiones}

La brecha actual en cirugía general y sus especialidades derivadas parece tener una leve inclinación hacia éstas últimas si comparamos el proporcional de los especialistas actualmente existentes. De éstas, la cirugía vascular y plástica representan $60 \%$ de la brecha de especialistas y destacan también por ser las con más inscritos en la SIS, pero con una matrícula limitada a Santiago y Concepción solamente. Si bien lo anterior es cierto, nominalmente se necesitan más cirujanos generales que sub-especialistas para satisfacer las necesidades de la población.

La coexistencia de un mercado público y privado, sumado a la escasa información respecto a los recursos humanos de este último, no permiten caracterizar adecuadamente la brecha global, lo que plantea la necesidad de profundizar el estudio en esta área.

Así mismo, la escasa información disponible sobre la tendencia de la matrícula de especialistas generales y derivados sumada a la reciente aparición de flujos migratorios aún no estudiados, dificulta la estimación del desarrollo del capital humano en esta área.

La convivencia entre las intenciones individuales y las necesidades generales no siempre es armoniosa, por lo que la gestión de la autoridad tanto a nivel central como a nivel local en las distintas instituciones de salud, tendrá un rol fundamental en el desarrollo de la especialidad, dejando espacios a futuro más allá de la formación universitaria, para resolver una urgente necesidad de nuestra población. Asimismo, la política de formación nacional deberá actualizarse en la medida que se avance en el cierre de brechas, para propender que la formación de capital humano especializado se ajuste a las necesidades reales del país.

Finalmente, consideramos que el presente trabajo muestra un interesante nicho de estudio aún poco desarrollado, pero de vital importancia, tanto para el desarrollo de la especialidad como para el beneficio de los usuarios de las atenciones de salud.

\section{Referencias}

1. Bruns SD, Davis BR, Demirjian AN, Ganai S, House MG, Saidi RF, et al. The Subspecialization of Surgery: A Paradigm Shift. J Gastrointest Surg 2014; 18 (8): 152331.
2. Cogbill TH, Klingensmith ME, Jones AT, Biester TW, Malangoni MA. Resident Preparation for Careers in General Surgery: A Survey of Program Directors. En: Journal of Surgical Education [Internet]. Elsevier; 2015. p. e251-7. Disponible en: http://dx.doi.org/10.1016/j. jsurg.2015.05.001.

3. Fakhry SM, Robinson L, Hendershot K, Reines HD. Surgical residents' knowledge of documentation and coding for professional services: an opportunity for a focused educational offering. Am J Surg. 2007.

4. Grover BT, Kothari SN. Fellowship Training: Need and Contributions [Internet]. Vol. 96, Surgical Clinics of North America. Elsevier Inc; 2016. p. 47-57. Disponible en: http://dx.doi.org/10.1016/j.suc.2015.09.003.

5. Banco Mundial, Ministerio de Salud. Estudio de brechas de oferta y demanda de médicos especialistas en Chile. 2009.

6. Ministerio de Salud. Informe de brechas sobre personal de salud por Servicio de Salud [Internet]. Subsecreta. Glosa 01, letra i. Ley de presupuesto año 2017. Santiago; 2017. Disponible en: http://web.minsal.cl/wp-content/ uploads/2015/08/Informe-Brechas-RHS-en-Sector-Público_Abril2017.pdf.

7. Ministerio de Salud. Informe sobre Brechas de Personal de Salud por Servicio de Salud [Internet]. Subsecretaría de Redes Asistenciales, editor. Glosa 01 letra i. Ley de presupuesto $\mathrm{N}^{\circ} 20.882$ y 20.890 año 2016. Santiago; 2016. 71 p. Disponible en: http://web.minsal.cl/wp-content/uploads/2015/08/Informe-Brechas-RHS-en-Sector-Público_Marzo2016.pdf.

8. Guillou M, Carabantes CJ, Bustos FV. Disponibilidad de médicos y especialistas en Chile. Rev Med Chile 2011; 139 (5): 559-70.

9. OECD. Health at a Glance 2017 [Internet]. OECD Indicators, editor. Paris: OECD Publishing; 2017. (Health at a Glance). Disponible en: http://www. oecd-ilibrary.org/education/education-at-a-glance-2017_eag-2017-en.

10. OECD. Health at a Glance 2015 [Internet]. OECD Indicators, editor. OECD Indicators. OECD Publishing; 2015. 218 p. (Health at a Glance). Disponible en: http:// www.oecd-ilibrary.org/social-issues-migration-health/ health-at-a-glance-2015_health_glance-2015-en.

11. Espinoza R, Danilla S, Valdés F, San Francisco I, Llanos O. Evaluación de los Programas de Formación en Cirugía General. Encuesta a los alumnos de postítulo. Rev Med Chile [Internet]. 2009; 137: 940-5. Disponible en: http://www.scielo.cl/scielo.php?script=sci_arttext\&pi$\mathrm{d}=$ S0034-98872009000700013 [citado el 20 de octubre de 2013].

12. Departamento de Formación Capacitación y Estudio. 
Memo C32 No50 Envía orientaciones respecto al Programa de formación directa de los Servicios de Salud. memo C32/50/2011 Santiago, Chile; 2011 p. 2.

13. Subsecretaria de Redes Asistenciales; Ministerio de Salud. Decreto 8. Reglamento de certificación de las especialidades y subespecialidades de los prestadores individuales de salud y de las entidades que la otorgan [Internet]. 1052275 Chile: Biblioteca del Congreso Nacional de Chile; 2013. Disponible en: https://www. leychile.cl/N?i=1052275\&f=2015-08-13\&p=. 